This is the last draft of the following paper. De Cruz, H. \& De Smedt, J. (2010). Paley's iPod: The cognitive basis of the design argument within natural theology. Zygon. Journal of Religion \& Science, Volume 45, Issue 3, pages 665-684, DOI: 10.1111/j.1467-9744.2010.01120.x

\title{
Paley's iPod: The cognitive basis of the design argument within natural theology
}

Helen De Cruz, Centre of Analytical Philosophy, Catholic University of Leuven, Kardinaal Mercierplein 2, 3000 Leuven, Belgium, (corresponding author)

Johan De Smedt, Department of Philosophy and Ethics, Ghent University, Blandijnberg 2, 9000 Gent, Belgium

Keywords: Argument from design; cognitive biases; design stance; inference to the best explanation; intelligent design; natural theology; probabilistic inference; teleological reasoning

\begin{abstract}
The argument from design stands as one of the most intuitively compelling arguments for the existence of a divine creator. Yet, for many scientists and philosophers, Hume's critique and Darwin's theory of natural selection have definitely undermined the idea that we can draw any analogy from design in artifacts to design in nature. Here, we examine empirical studies from developmental and experimental psychology to investigate the cognitive basis of the design argument. From this it becomes clear that humans spontaneously discern purpose in nature. When constructed theologically and philosophically correctly, the design argument is not presented as conclusive evidence for God's existence, but rather as an abductive, probabilistic argument. We examine the cognitive basis of probabilistic judgments in relationship to natural theology. Placing emphasis on how people assess improbable events, we clarify the intuitive appeal of Paley's watch analogy. We conclude that the reason why some scientists find the design argument compelling and others do not lies not in any intrinsic differences in assessing design in nature, but rather in the prior probability they place on complexity being produced by chance events or by a Creator. This difference provides atheists and theists with a rational basis for disagreement.
\end{abstract}

\section{Introduction}

For many scientists and philosophers, Hume's critique (1779) and Darwin's theory of natural selection (1859) have definitely undermined the idea that we can draw any analogy from design in artifacts to design in nature. Yet the argument from design remains one of the most popular arguments for God's existence. It enjoys an enduring appeal, going back as early as Plato's Timaeus, Cicero's De Natura Deorum and Aquinas' Summa Theologiae. Although it garnered particular attention around the turn of the 19th century, with Paley ([1802]2006) as the best-known example, recent formulations can be found in the work of Swinburne (e.g., 1968), Plantinga (e.g., 1991) and proponents of Intelligent Design. Some (e.g., Wattles 2006) have 
argued that the appeal of the design argument can be explained by Aristotle's pervasive influence on Western philosophy and theology in his concept of nature as inherently purposive. Although cultural factors undeniably played an important role, we propose that the popularity of the design argument runs deeper, and that its argumentative structure can be traced back to evolved properties of the human mind.

Recent discussions of the design argument in philosophy and theology (e.g., Robertson 2007, letter 6) have paid relatively little attention to the psychological underpinnings that lead to the understanding, acceptance or rejection of this argument. The aim of this paper is to investigate the cognitive basis of the design argument, drawing on empirical studies from developmental and experimental psychology. We focus on two aspects: the tendency of humans to discern teleology in nature, and the way they intuitively assess probabilities. A better understanding of these aspects not only elucidates the lasting popularity of the design argument, but can also help theists and atheists to construct a rational basis for disagreement. We begin by outlining an analysis of the epistemic properties of the design argument. Next, we examine the cognitive basis of teleological reasoning and the design stance in children and adults. We then discuss the probabilistic aspects of the design argument. Finally, we explore why theists and atheists disagree on the plausibility of the argument.

\section{The argument from design}

The argument for the existence of a divine creator, based on evidence of design in nature, has recently enjoyed a revival in theology and philosophy. Biologists (e.g., Dawkins [1986] 1991, 4-5) praise the argument for its explanatory coherence and intuitive appeal. Paley's image of the watch on the heath was certainly not the earliest formulation of the design argument, nor even of the watch analogy, but its familiarity makes it a suitable starting point. The argument Paley presented in Natural theology can be summarized as follows: if one encounters a watch, the complexity of this artifact and the interrelations of its parts lead to the inference that it is the product of purposive design. From this Paley concluded that complexity in nature is also the product of a Designer, because proposing that it could have been brought about by chance would be absurd (Paley [1802] 2006, 7-15).

This argument has interesting epistemic properties: it is both an analogy and an inference to the best explanation. Analogies map the structure of a well-known domain (the source domain) onto a lesser-known problem (the target domain). In distant analogies the structures of source and target domains greatly differ in their basic ontological properties. The design argument is a distant analogy in that it maps the artifactual domain (source domain) onto the natural world (target domain). Artifacts exhibit goal-directedness in their design: they are intentionally created by designers who had their function in mind. As organisms exhibit goal-directness in their design, they must also be the product of a purposeful designer. Although this analogical structure has often been attacked on the ground that it is inconclusive (e.g. Hume 1779, Frank 2004), it is worth noting that distant analogies are well-established in scientific practice as a way to gain insight into new problems. When the conceptual structure of the target domain is relatively unknown, as in the case of scientific discovery, scientists often resort to analogical reasoning of this sort as an epistemic action. Historical examples include Kepler's mapping of the properties of gravity onto the properties of light (i.e., the fact that sunlight dissipates with 
increasing distance between the Sun and the planets it is cast upon) to explain why planets further from the Sun move more slowly, in this case, caused by a weakening of the gravitational force with increasing distance from the Sun (Gentner et al. 1997), and Darwin's analogy of a hundred thousand wedges to examine the force of natural selection (Millman and Smith 1997). A more recent example is the Swiss army knife analogy as a way to conceptualize the evolved structure of the human brain in evolutionary psychology (Cosmides and Tooby 1995, 88). The analogical structure of the design argument is thus epistemic; it is performed to gain insight into an unfamiliar domain (God's creation). In many early versions, the design argument served a heuristic, rather than a strictly argumentative purpose, as in the works of $17^{\text {th }}$ century natural philosophers like Bernard Nieuwentijt and Antonie van Leeuwenhoek, who perceived design as a source of wonderment about nature.

Traditionally, design arguments had an inductive argumentative structure. They began with the empirical observation that all complex, functional objects of known origin were products of intelligent design. Then came the inductive step, in which one infers that what is true for some members of a class is true for all members. Hume (1779, part II, 56-60) dispensed with this way of reasoning by arguing that artifacts and biological organisms are too dissimilar to be classed together. When we see a house, we can reasonably infer that it has an architect or builder, because we know from experience that this particular effect flows from that particular cause. But we have no guarantee that the universe bears such a resemblance to a house as to invoke a Designer; we do not know in how far our analogy is reliable. Indeed, because an object resembles other objects in that it has property $P$ does not imply that it also resembles them in other respects. To suppose that it does is affirming the consequent, which is a logical mistake. Although Paley did not mention Hume explicitly, the structure of his watchmaker analogy escaped this criticism by adopting a different strategy. It is an inference to the best explanation (IBE), which has the following structure:

IBE Given evidence $E$ and a pool of plausible, potential explanations $H_{1}, \ldots$, $H_{n}$ of $E$, if $H_{i}$ explains $E$ better than any of the other hypotheses, infer that $H_{i}$ is closer to the truth than any of these others (Douven 2002, 359).

IBE enables us to probabilistically infer that a given hypothesis is closer to the truth than other hypotheses because it explains the available evidence better than rival explanations. In this probabilistic aspect, the design argument differs from deductive proofs for the existence of God, such as Anselm's ontological proof. IBE escapes Hume's critique, because it does not rely on induction, as it simply argues that there is no better explanation for order and complexity than design (Gliboff 2000). This fundamental probabilistic aspect of the design argument has received relatively little attention in the philosophical literature (but see Sober 2002). Having established apparent design and probabilistic inference as two key properties of the design argument, we will now examine the possible cognitive bases for its rational acceptability. 


\section{How we infer design}

\subsection{The design stance}

To Paley, the conclusion that a watch is purposefully designed was self-evident. Our perception of its interrelated parts, formed and adjusted to each other-the coiled elastic spring, the flexible chains, the cogwheels-each fashioned out of the material that suits their intended function best, should lead us to infer that the watch must have had a maker who formed it for a specific purpose. Yet Paley acknowledged that such seemingly spontaneous inferences require contextual knowledge about the artifact under consideration: 'it requires indeed an examination of the instrument, and perhaps some previous knowledge of the subject, to perceive and understand it' (Paley [1802] 2006, 8). In the case of the watch, Paley could infer the intent of the designer, as he was familiar with the class of artifacts to which the watch belongs. But what would happen if he pitched his foot against an unfamiliar object, such as an iPod? Would he have inferred design as automatically as in the case of the watch? Its sleek shape, carefully integrated buttons and intended function would have presented a puzzle to him. Unfamiliar artifacts can be so outlandish that people can be led to believe that they are not the work of human designers. During World War II, when the indigenous inhabitants of Papua New Guinea were first confronted with western goods from the American army, they believed that these were gifts of the ancestors. This led to the emergence of cargo cults, whose adherents are still trying to ritually lure airplanes into dropping more cargo, more western goods (Frank 2004). And what to think of people who observe UFOs? Often these alleged spaceships are no more than military reconnaissance airplanes, crashing weather balloons, or even bright planets. Nevertheless, people do infer that the objects are intentionally designed by supernatural agents and by extraterrestrials respectively. To gain a better understanding of the design argument, it is therefore useful to examine how humans infer design, what constitutes necessary and sufficient conditions for something to be a product of design, and how creator and artifact are causally linked.

Evidence from developmental psychology suggests that design is not a feature we can objectively infer. Remarkably, neither complexity nor order are necessary and sufficient conditions to decide whether an object is purposefully created. For example, Gelman and Ebeling (1998) showed two-year-olds a stain vaguely resembling a bear. They told some subjects that the spot was created accidentally, by kicking over a bottle of paint, whereas another group learned that the shape was painted intentionally. Only the children in the latter group called it 'a bear'. Thus the perception of the stain as representational or accidental depends critically on the prior information the children received on how it was brought about. In a similar experiment (Gelman and Bloom 2000), adults saw a variety of artifacts, but were given two diverging accounts of how the objects came into being. In the unintentional version a piece of cloth was accidentally caught in a machine, resulting in holes being punched at regular intervals. In the intentional version, a person took scissors and carefully cut holes at regular intervals. Subjects were more prone to call the object a 'belt' if they believed it was intentionally created. Apparently, design is in the eye of the beholder: our judgment that something is an artifact depends on our foreknowledge that the artifact was intentionally created. Once we are familiar with specific classes of artifacts, we can reasonably infer that a particular member of a class was created with the intention of belonging to this class. When we see a chair in 
a pile of rubbish, we conclude that the object was created to fulfill a specific function (to sit on) and to be of a specific class (chairs). This stance also provides useful inferences when we have to identify classes of non-utilitarian objects, such as ships in bottles: although these boats will never sail, we still call them ships, because the designer intended them to belong to this class of objects. The intimate relationship between design and intention was noted by Dennett (1987, 16-17) and Bloom (1996) who argue that humans take an intuitive design stance: we use the designer's intention to infer the class the object belongs to. It was on this inference that Paley relied in his assertion that the watch was a product of intentional design.

Conversely, knowledge of the designer's intention can help us to infer an object's intended function or identity. Take as an example Bloom and Markson's (1998) experiments in which three- and four-year-olds were shown featureless ovals that were purportedly drawn by a child with a broken arm that because of this could not draw well. The young subjects were told that these were drawings of chickens (three vertical ovals) and a pig (one horizontal oval). When prompted, the preschoolers effortlessly identified the pig, because they reasonably inferred that the artist would draw objects from the same category in the same way. Young children intuitively regard the creator of an artifact as having privileged knowledge about both its name and its intended function. This was illustrated by an experiment (Jaswal 2006) in which preschoolers saw objects that were given an anomalous label, e.g., the experimenter shows the child a key-shaped object and says 'you are not going to believe this, but this is actually a spoon'. Children were only willing to adopt the anomalous name if the experimenter referred to the object as something he created, not as something he merely found. Recognizing that the creator of an artifact has the prerogative to name it marks an important step in the development of the design stance.

The intended function and identity of an object thus inextricably link the creator with the created object. This aspect of the design stance is particularly interesting in the case of broken objects: although broken watches and fragile chairs cannot perform their intended function, we still name these objects watches and chairs because they were originally created to fulfill the intended function of their artifact class. When 9-year-olds and adults are presented with broken artifacts, they still label them according to their intended function, except if the transformation has changed the object beyond recognition (Gutheil et al. 2004). Paley voiced this intuition aptly when he stated: '...neither [...] would it invalidate our conclusion, that the watch sometimes went wrong, or that it seldom went exactly right. The purpose of the machinery, the design, and the designer, might be evident, and in the case supposed would be evident, in whatever way we accounted for the irregularity of the movement, or whether we could account for it or not. [...] If by the loss, or disorder, or decay of the parts in question, the movement of the watch were found in fact to be stopped, or disturbed, or retarded, no doubt would remain in our minds as to the utility or intention of these parts' (Paley [1802] 2006, 8-9).

The human propensity of inferring design may be due to the distinct evolutionary history of our species. Humans rely to a unique extent on tools for their survival. Whereas other primates use mostly unmodified objects as tools, archaeological evidence for stone-knapping in hominids goes back as far as 2.7 million years (Semaw 2000). By adopting the design stance, hominid children might 
have learned to use and fashion tools more efficiently. Indeed, comparative studies of social learning in children and chimpanzees reveal stark contrasts in the way new tools are used: whereas children take the intention of the person who demonstrates these tools as guidance, chimpanzees rely more extensively on the physical properties of the tools to figure out how they work (Horner and Whiten 2005). The design stance provides children with a useful heuristic to learn about their environment. It allows them to 'ignore the actual (possibly messy) details of the physical constitution of an object, and [relying on] the assumption that it has a certain design, predict that it will behave as it is designed to behave under various circumstances' (Dennett 1987, 16-17). Without the design stance, we would not possibly learn to use and name hundreds of tools and other artifacts, but would perhaps be limited to the less than ten tool types a typical community of wild chimpanzees entertains (Whiten et al., 1999). The hypothesis that the design stance is a product of natural selection, rather than the cultural product of Aristotelian teleology, finds support in the fact that it also occurs in nonwestern cultures, even those where material culture is relatively sparse, like the Shuar, an Andean Native American culture (German and Barrett 2005), and that infants and young toddlers rely on it to learn the names of novel objects and how to use them (Casler and Kelemen 2007).

\subsection{Intuitive teleology}

Whereas the design stance might have evolved for the purpose of rapidly categorizing and using artifacts, humans also possess a natural propensity for teleological reasoning. This propensity is most marked for biological entities, but it can apply to almost all categories of objects. Across cultures (see e.g., Barrett 2004), humans have the intuition that animals and plants possess adaptations that are self-beneficial, such as claws for defense or thorns for protection against being eaten. Young children, however, not only attribute purpose to artifacts and biological adaptations, but also to entire organisms (what are lions for? 'to go in the zoo') and nonliving natural kinds like clouds ('for raining') - a tendency termed 'promiscuous teleology' (Kelemen 2004). Moreover, when given a choice between teleological and non-teleological explanations, preschoolers and elementary school children prefer teleological accounts. For example, when asked whether rocks are pointy because of natural processes (e.g., 'bits of stuff piled up for a long period of time') or because of teleological functions (e.g., 'so that animals could scratch on them when they got itchy'), children typically endorse the latter (Kelemen 2003). At around ten to twelve years of age, the preference for teleological explanations lessens, probably because adolescents acquire elaborate coherent mechanistic explanations through schooling. Although mountains can be climbed, few adults would claim that mountains are there to climb on. This is because our learned knowledge that mountains are formed by tectonic activity or volcanism is incompatible with teleological explanations, where the function provides a sufficient reason for why the structure exists. Remarkably, patients with Alzheimer's disease show a re-emerging preference for teleological explanations. For example, they think that rain is there so that plants and animals could have water to drink and grow, rather than the acquired explanation that rain occurs by water condensing into clouds and forming droplets (Lombrozo et al. 2007). An increased tendency to teleology is also observed in people with little schooling such as Roma adults (Gypsies from central European descent). Formal education 
seems to reduce a preference for teleological explanations, but cannot eradicate them. Indeed, when educated adults are forced to make speeded judgments, they too, show a heightened acceptance of teleological explanations: when judging at a glance whether a statement is correct or not, they tend to endorse teleological, incorrect explanations, such as 'the Sun radiates heat because warmth nurtures life' (Kelemen and Rosset in press). Reasoning strategies observed in children persist into adulthood, but may be masked by secondary explanatory strategies. Once these become impaired (in the case of Alzheimer patients) or are unavailable (in the case of speeded judgments or lack of education), the intuitive, evolved strategies of childhood re-emerge. Although scientific education tends to lessen teleological reasoning, the tendency to apply teleology is not absent even among trained scientists. An analysis of the paleoanthropological literature (the study of human evolution) reveals that early theorists relied on extra-scientific intuitive explanatory strategies-until the late 1970s many paleoanthropologists thought that separate lineages of human races evolved toward an idealized endpoint (modern humans), a process denoted as orthogenesis, which is now uniformly rejected (De Cruz and De Smedt 2007).

Today, teleology is no longer considered a valid scientific principle in biology. It is hard to refrain from thinking that the eye's function is 'for seeing,' whereas it is more in tune with evolutionary theory to say that the eye's function can be described in terms of the effects it had for reproduction and survival in past organisms in which this structure evolved. Nevertheless, teleology is a useful heuristic to make sense of our everyday artifactual and biological environment - it enables us at a glance to see what a tool is for (e.g., a sharp edge for cutting) or to categorize animals as dangerous or not (e.g., presence of claws or fangs). As Kant had already argued in his Critique of Judgment, we use teleological explanations because the concept of purpose makes living things and artifacts more intelligible to us (Kant [1790] 1987, part II, § 66). The central place of teleology in human cognition can perhaps explain why it remains an important philosophical and theological principle, despite its lack of scientific plausibility. Note that the fact that our psychological propensities are sometimes at odds with scientific knowledge does not necessarily affect the rationality of our judgments. Not only are they often indispensable in everyday reasoning (our understanding of artifacts would be seriously compromised without the design stance), concepts like 'design' or 'purpose' would be meaningless without them. Whether or not such concepts are also scientifically meaningful, is a property that is to be empirically discovered.

\subsection{Are humans intuitive theists?}

Does the tendency to infer design also entail an inference to a Designer, as Paley and others have suggested? At this point, developmental and experimental psychological data do not present a unified picture. Lombrozo et al. (2007) found that although Alzheimer patients reasoned more teleologically, they were not more likely than healthy control subjects to invoke God as an explanation. In a study that probed Dutch primary school children's intuitive theories on the origin of species (Samarapungavan and Wiers 1997), the answers clustered together in different categories, including spontaneous generation, Lamarckism and pure essentialism (i.e., animals and plants have always existed). Although many children made teleological 
inferences, only about $10 \%$ made explicit reference to God or intelligent design. On the other hand, a comparable experiment by Evans (2001) in the United States, found that the majority of 10-year-olds endorsed creationist accounts of the origin of species, regardless of their religious background. Kelemen and diYanni (2005) obtained comparable results with British elementary school children, although the percentage of creationist accounts was significantly lower than with American subjects.

Several possible explanations might account for these findings. A strong position holds that humans are intuitive theists. In this view, creationism is a natural mode of reasoning which is only altered when children acquire explicitly nonreligious beliefs from their cultural environment. Bering (2006) defends this position, arguing that religious beliefs are biological adaptations that were directly selected to enhance cooperation, altruism and group cohesion. A weaker position (e.g., Bloom 2007) holds that religious belief itself is not innate but a byproduct of other cognitive adaptations such as agency detection and theory of mind. In this view, children acquire culturally transmitted religious beliefs easily because these key in on evolved propensities of the human mind. Here the step from design to Designer is not automatically made, but needs to be made explicit, as Paley and others in fact did. Support for this latter view comes from experiments where Hindu (Barrett 1998) and Christian (Barrett \& Keil 1996) college students had to recall stories about God. In doing so, they unconsciously distorted the stories to fit God into intuitive expectations they had about normal people, such as only attending to one person or one event at the same time. This indicates that representing an omniscient, omnipresent being is cognitively demanding and that expectations about normal agents structure reasoning about divine agents. Another view, suggested by Taede Smedes as he read this paper, holds that intuitive theism might be an evolved module, but that it depends on external cultural circumstances for its development, in the same way as the language faculty requires appropriate linguistic input to develop properly. It is our belief that the experimental evidence does not support the view that there is an intuitive theism. What is still required is an assignment of a probability to the existence of a Designer. In the next section, we will look in more detail at the probabilistic aspects of the design argument.

\section{Intuitive probability: Can chance events produce order and complexity? 4.1 The Annales and the Boeing}

Butler (1736) already observed that all human reasoning is probabilistic: as we are finite beings with knowledge that is restricted in time and space, we cannot claim absolute knowledge. From imperfect observations, we regularly draw far-reaching conclusions. Humans are naturally endowed with the ability to detect statistical frequencies in their environment (for a comprehensive overview, see De Cruz in press). This ability is not restricted to humans; it occurs in a wide variety of animal species, including those with relatively simple nervous systems like bumblebees (Real 1991). Human infants use probability inference to learn about their environment, such as the statistical detection of recurring sound patterns to chunk streams of continuous speech into words, which is crucial for word learning (Aslin et al. 1998). The design argument draws on our evolved ability to assess posterior probability, i.e. the probability that is assigned after the relevant evidence is taken into account. From 
the age of five onward, humans are fairly accurate in making such assessments (e.g., Girotto and Gonzales 2008). For example, if preschoolers are shown that more red than green chips are placed in a bag, they will correctly state that there is a higher chance that the experimenter will draw a red chip. However, if the experimenter says 'I can feel that the chip in my hand is round' and proportionally more round chips are green, children will update their probabilistic judgments in favor of green.

How likely is it that the apparent design in nature was intentionally created or, alternatively, that it happened by chance? Early proponents of the design argument have taken their intuition that chance does not produce order as a starting point: ' $\mathrm{He}$ who believes this may as well believe that if a great quantity of the one-and-twenty letters $[\ldots]$ were thrown upon the ground, they would fall into such order as to legibly form the Annales of Ennius. I doubt whether fortune could make a single verse of them. How, therefore, can these people assert that the world was made by the fortuitous concourse of atoms [...]' (Cicero $45 \mathrm{BC}$, book 2, $\S \mathrm{XXXVII).} \mathrm{Cicero}$ discarded the atomists' idea that chance collisions of elementary building blocks (atoms) formed the material world on the basis that chance has a low probability of producing order. Assuming that the 21 letters of the Roman alphabet are equally distributed into his 'great quantity', the chance of the first letter falling in the correct place is $1 / 21$, the chance that the first two letters are correct is thus $1 / 21 \times 1 / 21=$ $1 / 441$ (if the space is also treated as a letter, it would be $1 / 22 \times 1 / 22=1 / 484$ ). The chance that the letters would produce the approximately 7000 characters of the 600 lines that survive of Ennius' Annales (a now fragmentary epic poem on the history of Rome) is vanishingly small, being $1 / 21^{7000}$. Cicero's intuition has been re-iterated by countless other writers, including astronomer Fred Hoyle's image of hurling around scrap metal at random and happening to assemble a Boeing 747. Although all arrangements of the scrap metal are, with hindsight, equally improbable, very few of them will fly; similarly, although all combinations of 21 letters are equally unique, very few of them will produce a legible text, let alone the Annales. Dembski (1998) has developed this inference as the basis for his defense of Intelligent Design. Although it is intuitively compelling, rejecting chance as an explanation for complexity and design is problematic because, as Sober (2002) notes, there is no probabilistic equivalent of modus tollens, in other words we cannot state that

If hypothesis $H$ were true, observation $O$ would be highly improbable But $O$

Therefore, $H$ is not true

The lottery paradox aptly illustrates this: assume a fair lottery in which only 1 of 1000 tickets is the winner, therefore the probability of winning the lottery is very low. Yet winning the lottery does not cast doubt on its fairness. The law of likelihood in statistics stipulates that it is not the absolute value of the probability of data under a single hypothesis that is to be considered, but rather how the probability values compare under different hypotheses. The intuitive idea that improbability strengthens the existence of God is problematic in that it tacitly relies on an analogy between human and divine agency. In the case where we have to decide whether human design or chance is responsible, we rely on empirical knowledge of what human agents in fact do. In an example from Himma (2005), adapted from Dembksi (1998), 
suppose a political candidate's name appears first on the lists of voting ballots 40 out of 41 times. The probability of such an event occurring by chance is very small. But when we decide that a county clerk rigged the list, we rely on two pieces of tacit knowledge, namely that undecided voters are more likely to choose the first on the list, and that the county clerk wants a particular party to win. Being an intelligent agent, it is not unlikely that he rigged the list. We also know of cases where voting ballots were tampered with to win an election. Hence the hypothesis that the name was placed first 40 out of 41 times by design, rather than mere chance becomes very plausible indeed. But in the case of divine action, we do not have empirical knowledge to draw upon, thus no prior assumptions can be drawn about what God would or would not do. It is hence not possible to accord prior probabilities to the existence of God on the basis of empirical evidence. Without the necessary background data to make the design argument an IBE, this argument tacitly relies on an analogy between human and divine agency. Again, this version of the design argument becomes an argument from analogy-as we saw in section 2, this was successfully attacked by Hume, and it was precisely for this reason that Paley had recast the argument from design into an IBE.

\subsection{Probability and inference to the best explanation}

The reliability of IBE as an abductive strategy depends on the amount and quality of the data and the relevance of the data to the conclusion. If insufficient evidence is available, IBE may well lead us to choose 'the best of a bad lot' (van Fraassen, 1989, 143). In fact, the bad lot argument even applies if one has all the possible evidence as one may simply have failed to conceive of the true theory with this evidence in hand. If each letter that falls correctly is selectively retained, we need at most $21 \times 7000$ trials to complete what is now left of the Annales. Cicero, being unacquainted with the principle of cumulative selective retention, did not envision this possibility. Dawkins ([1986] 1991, 46-48) uses a similar analogy to illustrate this point: whereas one monkey could not possibly type a sentence from Hamlet, selective retention of keystrokes by many typing monkeys would solve the problem. Unfortunately, when using an IBE strategy, most modern versions of the design argument do not take natural selection and its principle of cumulative selective retention into account as a viable explanation. Dembski (1998), for example, holds that regularity, chance and design exhaust the possibilities, thereby sidestepping the combination of chance and regularity that is natural selection. To be sure, in 1802 natural selection was not in the pool of possible explanations. However, as Gliboff (2000) demonstrates, Paley did have a range of alternative materialist explanations, of which we will mention three. First, necessity: as everything has to have some form, it may as well be the present form, e.g., the eye is the actual realization of the possible ways to fill an eye socket. Second, he considered infinite trial and error: given an infinite time and universe, every possible configuration of matter could be produced, some of which turned out to be viable life-forms that persisted and reproduced, an interesting precursor to the concept of natural selection. Third, he discussed the claim that parts of organisms could arise before their function was determined, an exaptation theory avant la lettre (Paley [1802] 2006, p. 38-41).

These alternatives were being explored and hotly debated in Paley's time by early evolutionists like Buffon, Diderot and d'Holbach; Paley seems familiar with 
these authors as he mentioned Buffon explicitly and others implicitly. Next to these, he briefly discussed the special biological forces or organizing principles proposed by the Göttingen school of German biologists, such as Blumenbach, Kielmeyer and Reil (Paley [1802](2006), 218-225). Although rejected now, their Newtonian approach to biology in which they stipulated forces acting on biological entities (analogous to physical forces acting on physical entities) was conceivable and widely accepted at the time. The idea proposed by atomists like Democritos and Lucretius that very improbable things may happen in an infinite universe has its modern statistical formulation in Diaconis and Mosteller (1989, 859), in that 'with a large enough sample, any outrageous thing is likely to happen.' What led Paley to reject these alternatives? His answer was that the purported natural propensities required intelligent design: 'I am unwilling to give to it the name of an atheistic scheme [...] because, so far as I am able to understand it, the original propensities and the numberless varieties of them [...] are, in the plan itself, attributed to the ordination and appointment of an intelligent and designing Creator (Paley [1802] 2006, 224225).' One could dismiss this as a circular argument in that he rejected naturalistic explanations because they point to a Designer, the proposition that had to be proven. We want to argue that it can likewise be seen as arising out of the high probability Paley accorded to the existence of God-as we will argue in the next section, the likelihood of data can only be meaningfully assessed in relationship with hypotheses, which are accorded a prior probability.

\section{A rational basis for disagreement}

If humans are prone to discern design and teleology in nature, why then do some find the design argument more compelling than others? This may be due not to intrinsic differences in the way design and teleology are discerned, but to differences in the prior probability people place on the existence of a Designer. An interesting way to approach this problem is through an examination of how humans regard coincidences. In Griffiths' and Tenenbaum's view (2007), an event is a coincidence if it is judged to have a lower probability of occurring under our current theory of how the world works than under an alternative hypothesis. Coincidences play an important epistemic role in scientific discovery: the meteorologist Alfred Wegener noted that the coastlines of West-Africa and South-America fitted into each other like a jigsaw, that their geological strata matched, and that the distribution of species on both sides of the Atlantic was highly correlated. He thought that this pattern was not a mere coincidence, but that these continents were once joined and had drifted apart. Similarly, in the $19^{\text {th }}$ century, the physician John Snow noted that cholera outbreaks in London tended to cluster at public water pumps and inferred that this was not a coincidence, but provided evidence for his new theory that cholera was transmitted through polluted water (rather than bad air, the then favored theory). These examples suggest an intimate connection between coincidence and evidence. A mere coincidence occurs when the likelihood ratio in favor of an alternative theory is insufficient to overwhelm the prior odds against it. A coincidence becomes evidence when the likelihood ratio in favor of an alternative theory overcomes the prior odds against it, and leads us to accept that alternative theory. Because people differ in the prior probabilities they assign to alternative hypotheses, what is a coincidence to one person can be considered compelling evidence by another. 
In the case of the design argument, the competing hypotheses are $\mathrm{H}_{\text {mat }}$ (purposive and complex structures arose strictly through natural, material causes) and $\mathrm{H}_{\text {deo }}$ (design as the result of a Designer). In the framework of $\mathrm{H}_{\text {mat }}$, the occurrence of ordered complexity and apparent design presents a coincidence. Given that chance events tend to produce disorder, their probability is extremely low. Darwin's theory of natural selection has successfully solved this dilemma, because it relies on a combination of chance and law-like processes. Indeed, there is no other naturalistic theory that can explain why living things are improbably complex, why the interrelationships between their parts are highly functional and why they exhibit features that enhance their probability of surviving and reproducing in their environment. Proponents of $\mathrm{H}_{\text {mat }}$ can find epistemic justification in Darwin's explanation of design. To justify why they favor their view rather than $\mathrm{H}_{\text {deo }}$, they can cite examples of maladaptedness, and appeal to ontological parsimony as their explanation is restricted to observable, causal physical processes. In $\mathrm{H}_{\text {deo }}$ the occurrence of design is not improbable, as this theory explicitly proposes a Designer who made the universe orderly and purposeful. Under these epistemic circumstances, but not under $\mathrm{H}_{\text {mat }}$, design in nature becomes corroborative evidence for the existence of a Creator. Next to this, natural theologians can also appeal to ontological parsimony, since it reduces many kinds of explanation to one under $\mathrm{H}_{\text {deo }}$ (e.g., Swinburne 1968).

This model of prior probabilities can explain three puzzling facts. First, it explains why evolutionary thinkers writing before 1859 did not accept natural theology's design argument. Even in Paley's time, not everyone was led to accept $\mathrm{H}_{\text {deo }}$ although the arguments in favor of natural theology were widespread. Early evolutionists like Erasmus Darwin and biologists of the Göttingen school sought to describe biological forces that could assemble complexity in the same way as Newton had done for mechanics. These authors had a strong commitment to a physicalist worldview leading them to adopt the view that $\mathrm{H}_{\text {deo }}$ was unlikely, even though they did not have a compelling causal explanation for the apparent design. Second, it can explain why the design argument, despite its intuitive appeal, fails to convince nonbelievers. As long as plausible naturalistic explanations for design in nature are available, the design argument will fail to overturn their prior beliefs. Third, it may elucidate why well-established scientific data do not convince believers of design of the opposite. This is shown paradigmatically in the USA, where everyone has access to scientific education, yet creationism/Intelligent Design is widespread, and people have a severe distrust of evolutionary theory (see Miller et al. 2006). The epistemic force of the prior probabilities we accord to competing hypotheses is an important element in scientific and other formalized ways of reasoning. It can explain, for example, why scientists are unwilling to let go of a cherished theory even in spite of overwhelming evidence against it, as already described by Kuhn (1962). For theists, design in nature provides compelling circumstantial evidence for the existence of a Creator. Take as an illustration the Thomistic tradition, which emphasizes the role of understanding and knowledge (scientia) in belief. In this view, a successful natural theology would start out from self-evident premises, proceed by valid arguments and reach the conclusion that there is a person such as God (Plantinga 1991). As we have seen in section 3, humans are prone to discern design and teleology in nature. Within the epistemic context of $\mathrm{H}_{\text {deo }}$, the perceived design in nature that is a universal feature 
of human cognition can be taken as a self-evident premise from which the existence of a Creator can be argued. It is however, not a standalone argument that can convince those who do not believe in God (see also Himma 2005), especially since plausible naturalistic explanations have become available.

\section{Is there still a place for the design argument?}

Undeniably, the power of the design argument as an inference to the best explanation has been seriously weakened since Darwin and Wallace independently came up with natural selection as a naturalistic explanation for design. Given that the combination of random events and selective retention can explain most of the apparent design around us, can theologians still reasonably invoke design? Misrepresenting or altogether neglecting natural selection is the strategy most commonly adopted by proponents of Intelligent Design. Intelligent Design, however, is not a very desirable position for theologians to take because it makes scientific claims that need to be evaluated by scientific standards. As a scientific research program, it fails because its hypothesis of a Designer is too vague and too general to count as a scientific hypothesis; it cannot be used as a basis for empirical testing. Even concepts like irreducible complexity are too broad and too vacuous to be investigated by biologists - to date, there are no satisfying models of complexity that allows for investigation by empirical, quantitative methods. Moreover, using scientific standards to argue for divine action is a category mistake, since God is not an immanent cause like other natural causes (Smedes 2008). The most productive way for theologians and scientists to look at the argument from design is therefore to treat it as a metaphysical, rather than a scientific principle. Within this perspective, we think there are at least two cases in which a design position is still defensible.

A first case is presented by a position that endorses evolutionary biology but argues that God intervenes occasionally to fashion structures that could not have arisen through natural selection. In this position, one endorses intelligent design as a philosophical position but not Intelligent Design as a scientific research program, which conceptualizes evolution and design as two competing scientific explanations. Theologically, it follows a distinction that is commonly made between God's general actions (which pertain to the universe as a whole, and can be seen in the laws that govern physical, chemical and biological processes) and special actions (which include actions that lie beyond the normal physical processes). Whereas natural selection and other evolutionary processes belong to the former category, occasional design or intervention in these belongs to the latter. Johnson and Potter (2005), for example, argue that human natural language might be the product of purposive creation. They base their argument on the fact that adaptationist explanations require a plausible reason why the adaptation evolved. Adaptations evolve in response to specific selective pressures; they enhance the survival and reproduction of their bearers. For language, there are as yet no convincing adaptationist explanations: currently we do not know what language is an adaptation for, nor how and when it evolved. Despite the proliferation of adaptationist stories on the origin of language, such as social grooming, technological intelligence, cooperative hunting and sexual selection, none of these hypotheses has been able to substantiate itself into a theory. This leads Johnson and Potter (2005) to infer to the best explanation that purposive design brought language into being. Note that this position is distinct from Intelligent 
Design in that they explicitly endorse evolutionary theory as the best explanation for complexity in the living world.

A second, perhaps stronger case (because it does not rely on a God of the gaps) is found in scientists and theologians who regard design and evolution as complementary rather than as mutually exclusive explanatory frameworks. Watchmakers do not build watches from scratch; rather, they rely on the gradually accumulated innovations in time-keeping technology, which we can trace back from sundials and water-clocks, over the introduction of the spring to the modern digital watch. Upon close scrutiny, very few inventions appear de novo; most are the result of a gradual and cumulative retention of favorable variations (Basalla 1988). For example, the streamlined design of Polynesian canoes which is close to optimum can be traced through archaeological and historical data as the gradual and unconscious retention of favorable variations, with the perilous ocean as the selecting agent (Rogers and Ehrlich 2008). These insights on the origin of artifacts can be extended to divine design. The $19^{\text {th }}$-century botanist Asa Gray defended the view that natural selection is an 'a-fortiori extension to the supposed case of a watch which sometimes produces better watches, and contrivances adapted to successive conditions, and so at length turns out a chronometer, a town clock, or a series of organisms of the same type' (Gray 1888, 57). To take a recent example of this position, cell biologist Kenneth Miller ([1999] 2007) argues that God has initiated natural selection and other natural evolutionary processes as an indirect way to create complexity and design. For Miller ([1999] 2007, 213, 238, 253), the undetermined nature of evolution through natural selection and other natural processes enabled the evolution of truly free, truly independent beings. Similarly, Theodosius Dobzhansky, one of the founding fathers of the modern synthesis, wrote that ' $[t]$ he organic diversity becomes [...] reasonable and understandable if the Creator has created the living world not by caprice but by evolution propelled by natural selection [...] Evolution is God's or Nature's, method of Creation' (Dobzhansky 1973, 127). This position is stronger than Intelligent Design, because proponents of the latter-who see natural and supernatural causes as competing explanations for complexity in the living world-in many cases need to acknowledge that natural selection is the better explanation. This problem is avoided when one allows for the possibility that evolution and design are not mutually exclusive.

On the basis of modern evolutionary theory it is not possible to reject either $\mathrm{H}_{\text {mat }}$ or $\mathrm{H}_{\mathrm{deo}}$. Both positions depend on prior probabilities that are not assigned on the basis of scientific evidence but on the basis of metaphysical principles. It is interesting to note that both positions-physicalism and naturalistic theism-already existed in the earliest stages of evolutionary theory. Whereas Charles Darwin and Thomas Huxley did not admit room for God in their explanatory frameworks, Alfred Wallace and Asa Gray were theists, who treated divine action as complementary with a scientific worldview, not as a competitor. Wallace, for example, while continuing to endorse natural selection as the chief principle guiding the evolution of plants and animals, invoked intelligent design for the human mind: " $t \mathrm{t}$ ]he brain of pre-historic and of savage man seems to me to prove the existence of some power, distinct from that which has guided the development of the lower animals through their evervarying forms of being' (Wallace, 1871, 343). Fichman (2001) aptly argues that Wallace's theism, rather than a volte-face, was an integral part of his evolutionary 
thinking. Today, both schools of thought continue to exist side by side, with Richard Dawkins and Daniel Dennett as examples of strict materialists and Kenneth R. Miller and Simon Conway Morris as proponents of theistic evolution.

In this paper, we have argued that the argumentative structure of the design argument can be traced back to evolved biases of the human brain. It relies on the design stance, which leads us to treat complex and purposive structures as the product of design, and on intuitive teleology, the propensity of humans to discern purpose in nature. These cognitive biases are universal, although they can be masked by formal education or strengthened by religious upbringing. The step from design to Designer is perhaps more explicit, and relies on an inference to the best explanation. The plausibility of this inference relies on the prior probability one places on the existence of God. By making these differences in prior probability more explicit, theists (natural theologians, biologists and philosophers) and physicalist scientists and philosophers have a rational basis for disagreement.

\section{Acknowledgements}

Elements of this paper were presented at a colloquium on evolutionary theory and Intelligent Design, Brussels, Belgium, 20-21 August 2008, jointly organized by Ghent University and the Belgian Institute of Natural Sciences. We wish to express our gratitude to Igor Douven and Taede Smedes for their elucidating comments and suggestions to an earlier version of this paper, and to the anonymous reviewers for their helpful suggestions. This research is supported by grant 3H070815 from the Research Foundation Flanders and grant COM07/PWM/001 from Ghent University.

\section{References}

Aslin, Richard N., Jenny R. Saffran, and Elissa L. Newport. 1998. "Computation of conditional probability statistics by 8-month-old infants." Psychological Science 9: 321-324.

Barrett, H. Clark. 2004. "Descent versus design in Shuar children's reasoning about animals." Journal of Cognition and Culture 4: 25-50.

Barrett, Justin L. 1998. "Cognitive constraints on Hindu concepts of the Divine." Journal for the Scientific Study of Religion 37: 608-619.

Barrett, Justin L. and Frank C. Keil. 1996. "Conceptualizing a nonnatural entity: Anthropomorphism in God concepts." Cognitive Psychology 31: 219-247.

Basalla, George. 1988. The evolution of technology. Cambridge: Cambridge University Press.

Bering, Jesse M. 2006. "The folk psychology of souls." Behavioral and Brain Sciences 29: 453-462.

Bloom, Paul. 1996. "Intention, history and artifact concept." Cognition 60: 1-29.

—. 2007. "Religion is natural." Developmental Science 10: 147-151.

Bloom, Paul and Lori Markson. 1998. "Intention and analogy in children's naming of pictorial representations." Psychological Science 9: 200-204.

Butler, Joseph. 1736. The analogy of religion, natural and revealed, to the constitution and cause of nature. Retrieved on 8 August 2008 from http://posner.library.cmu.edu/Posner/books/book.cgi?call=219_B98A_1736 
Casler, Krista and Deborah Kelemen. 2007. "Reasoning about artifacts at 24 months: The developing teleo-functional stance." Cognition 103: 120-130.

Cicero, Marcus T. 45 BC. De natura deorum. Retrieved on 5 August 2008 from http://trisagionseraph.tripod.com/Texts/Cicero2.html

Cosmides, Leda, \& Tooby, John. 1995. "Beyond intuition and instinct blindness: Toward an evolutionarily rigorous cognitive science." In Cognition on cognition, by Jacques Mehler and Susana Franck, 69-105. Cambridge, Ma.: MIT Press.

Darwin, Charles. 1859. On the origin of species by means of natural selection or the preservation of favoured races in the struggle for life. London: John Murray.

Dawkins, Richard. [1986] 1991. The Blind Watchmaker. London: Penguin.

De Cruz, Helen and Johan De Smedt. 2007. "The role of intuitive ontologies in scientific understanding-The case of human evolution." Biology and Philosophy 22: 351-368.

De Cruz, Helen. in press. "The role of intuitive probability in scientific theory formation." In Foundations of the Formal Sciences VI: Reasoning about probabilities and probabilistic reasoning, by Benedikt Löwe, Eric Pacuit, and Jan-Willem Romeijn.

Dembski, William A. 1998. The design inference: Eliminating chance through small probabilities. Cambridge: Cambridge University Press.

Dennett, Daniel (1987). The intentional stance. Cambridge, Ma.: MIT Press.

Diaconis, Persi and Frederick Mosteller (1989). "Methods for studying coincidences." Journal of the American Statistical Association 84: 853-861.

Dobzhansky, Theodosius. 1973. "Nothing in biology makes sense except in the light of evolution." The American Biology Teacher March: 125-129.

Douven, Igor. 2002. "Testing inference to the best explanation." Synthese 130: 355377.

Evans, E. Margaret. 2001. "Cognitive and contextual factors in the emergence of diverse belief systems: Creation versus evolution." Cognitive Psychology 42: 217-266.

Fichman, Martin. 2001. "Science in theistic contexts: A case study of Alfred Russell Wallace on human evolution. Osiris 16: 227-250.

Frank, Patrick. 2004. "On the assumption of design." Theology and Science 2: 109130.

Gelman, Susan A. and Paul Bloom. 2000. "Young children are sensitive to how an object was created when deciding what to name it." Cognition 76: 91-103.

Gelman, Susan A. and Karen S. Ebeling. 1998. "Shape and representational status in children's early naming." Cognition 66: 35-47.

Gentner, Derdre, Sarah Brem, Ronald W. Ferguson, Arthur B. Markman, Björn B. Levidow, Philip Wolff, and Kenneth D. Forbus. 1997. "Analogical reasoning and conceptual change: A case-study of Johannes Kepler." Journal of the Learning Sciences 6: 3-40.

German, Tim P. and H. Clark Barrett. 2005. "Functional fixedness in a technologically sparse culture." Psychological Science 16: 1-5.

Girotto, Vittorio and Michel Gonzales. 2008. "Children's understanding of posterior probability." Cognition 106: 325-344. 
Gliboff, Sander. 2000. "Paley's design argument as an inference to the best explanation, or, Dawkins' dilemma." Studies in History and Philosophy of Biological \& Biomedical Science 31: 579-597.

Gray, Asa. 1888. Darwiniana: Essays and reviews pertaining to Darwinism. New York: Appleton.

Griffiths, Thomas L. and Joshua B. Tenenbaum. 2007. "From mere coincidences to meaningful discoveries." Cognition 103: 180-226.

Gutheil, Grant, Paul Bloom, Nohemy Valderrama, and Rebecca Freedman. 2004. "The role of historical intuitions in children's and adults' naming of artifacts." Cognition 91: 23-42.

Himma, Kenneth. 2005. "The application-conditions for design inferences: Why the design arguments need the help of other arguments for Gods existence." International Journal for Philosophy of Religion 57: 1-33.

Horner, Victoria and Andrew Whiten. 2005. "Causal knowledge and imitation/emulation switching in chimpanzees (Pan troglodytes) and children (Homo sapiens)." Animal Cognition 8: 164-181.

Hume, David. 1779. Dialogues concerning natural religion, second edition. London: Hafner.

Jaswal, Vikram K. 2006. "Preschoolers favor the creator's label when reasoning about an artifact's function." Cognition 99: B83-B92.

Johnson, Jeffery L. and Joyclynn Potter. 2005. "The argument from language and the existence of God." The Journal of Religion 85: 83-93.

Kant, Immanuel. [1790] 1987. Critique of Judgment (translated by Werner Pluhar). Indianapolis: Hacket.

Kelemen, Deborah. 2003. "British and American children's preferences for teleofunctional explanations of the natural world." Cognition 88: 201-221.

—. 2004. "Are children "intuitive theists"? Reasoning about purpose and design in nature." Psychological Science 15: 295-301.

Kelemen, Deborah and Cara DiYanni. 2005. "Intuitions about origins: Purpose and intelligent design in children's reasoning about nature." Journal of Cognition and Development 6: 3-31.

Kelemen, Deborah and Evelyn Rosset. in press. "The human function compunction: Teleological explanation in adults." Cognition.

Kuhn, Thomas S. 1962. The Structure of Scientific Revolutions. Chicago: Chicago

University Press.

Lombrozo, Tania, Deborah Kelemen, and Deborah Zaitchik. 2007. "Inferring design: Evidence of a preference for teleological explanations in patients with Alzheimer's disease." Psychological Science 18: 999-1006.

Miller, Kenneth R. [1999] 2007. Finding Darwin's God: A scientist's search for common ground between God and evolution. New York: Harper.

Miller, Jon, Eugenie Scott and Shinji Okamoto. 2006. "Public acceptance of evolution." Science 313: 765-766.

Millman, Arthur B. and Carol L. Smith. 1997. "Darwin's use of analogical reasoning in theory construction." Metaphor and Symbol 12: 159-187. 
Paley, William. [1802] 2006. Natural theology or evidence for the existence and attributes of the Deity, collected from the appearances of nature. Oxford: Oxford University Press.

Plantinga, Alvin. 1991. "The prospects for natural theology." Philosophical Perspectives 5: 287-315.

Real, Leslie A. 1991. "Animal choice behavior and the evolution of cognitive architecture." Science 253: 980-986.

Robertson, David. 2007. The Dawkins letters. Challenging atheist myths. Fearn: Christian Focus.

Rogers, Deborah S. and Paul R. Ehrlich. 2008. "Natural selection and cultural rates of change." Proceedings of the National Academy of Sciences USA 105: 34163420 .

Samarapungavan, Ala and Reinout W. Wiers. 1997. "Children's thoughts on the origin of species: A study of explanatory coherence." Cognitive Science 21: 147-177.

Semaw, Sileshi. 2000. "The world's oldest stone artefacts from Gona, Ethiopia: Their implications for understanding stone technology and patterns of human evolution between 2.6-1.5 million years ago." Journal of Archaeological Science 27: 1197-1214.

Smedes, Taede A. 2008. "Beyond Barbour or back to basics? The future of scienceand-religion and the quest for unity." Zygon: Journal of Religion and Science 41: 445-464.

Sober, Elliott. 2002. "Intelligent design and probability reasoning." International Journal for Philosophy of Religion 52: 65-80.

Swinburne, Richard G. 1968. "The argument from design.” Philosophy 43: 199-212.

van Fraassen, Bas. 1989. Laws and Symmetry. Oxford: Clarendon Press.

Wallace, Alfred R. 1871. Contributions to the theory of natural selection. London: Macmillan.

Wattles, Jeffrey 2006. "Teleology past and present." Zygon: Journal of Religion and Science 41: 445-464.

Whiten, A., J. Goodall, W.C. McGrew, T. Nishida, V. Reynolds, Y. Sugiyama, C.E.G. Tutin, R.W. Wrangham, and C. Boesch. 1999. "Cultures in chimpanzees." Nature 399: 682-685. 